\title{
Descrição da genitália masculina de algumas espécies de Agelaia Lepeletier 1836 (Vespidae, Epiponini) \\ Ruyter Jesus de Cerqueira ${ }^{1}$ e Sergio Ricardo Andena ${ }^{2}$
}

1.Bolsita Probic, Graduando no curso de Ciências Biológicas,Universidade Estadual de Feira de Santana,e-mail: ruyter1994@gmail.com

2.Orientador, Departamento de Ciências Biológicas, Universidade Estadual de Feira de Santana,e-mail: sergioricardoandena@gmail.com

\section{Palavras chave: Epiponini, genitália de macho, Agelaia}

\section{Introdução:}

Agelaia conta atualmente com 31 espécies descritas que se distribuem do México ao norte da Argentina (Cooper, 2001), 15 delas ocorrem no Brasil, sendo uma endêmica. Uma espécie fóssil também foi registrada para esse gênero (Carpenter \& Grimaldi, 1997). A diferenciação de castas para Agelaia é a mais pronunciada dentre os Epiponini, com clara diferenciação entre rainhas e operárias (Jeanne1991). Os ninhos são variáveis, alguns dentre os maiores já descritos para os Epiponini, com população de até um milhão de indivíduos (Richards \& Richards, 1951; Richards, 1978; Zucchi et al., 1995; Wenzel, 1998). A genitália masculina de Epiponini tem sido de fundamental importância para elucidação das relações entre as espécies de vários gêneros dessa tribo. Dessa forma a presente proposta visa a descrição/redescrição da genitália de algumas espécies de Agelaia. Os machos disponíveis tiveram suas genitálias retiradas e clareadas. Um total de quatro espécies tiveram suas genitálias descritas (Agelaia angulata, Agelaia areata, Agelaia cajennensis, A. centralis).

Infelizmente, dados sobre a genitália de machos em grupos de Epiponini, ainda são raros na literatural, devido, como é de se esperar de grupos haplodiplóides, a dificuldade de se encontrar machos em colônias de vespas sociais. Varios fatores tem sido descritos como importantes para o grupo como: a relação entre o comprimento e a largura do parâmero; o espinho do parâmero, bem como a presença ou ausencia de cerdas; o edeago, que em Agelaia não apresentou variações, exceto em relação aos dentes, que podem ser uniformes, maiores ou menores no ápice ou na base. $\mathrm{O}$ cuspis e o digitus das espécies não apresentaram diferenças dignas de nota, mas podem apresentar pequenas variações na região apical, sendo mais arredondada ou pontiaguda, bem como o tamanho e distribuição das cerdas.

Os dados apresentados serão de importância taxonômica, bem como na elucidação das relações entre as espécies do gênero. 


\section{Referências}

Andena, S.R., F. B. Noll, J.M. Carpenter and R. Zucchi. 2007. Phylogenetic analysis of the neotropical Pseudopolybia de Saussure, 1863, with description of the male genitalia of Pseudopolybia vespiceps (Hymenoptera, Vespidae, Epiponini). American Museum Novitates 3486: -11 .

Carpenter, J.M. and D.A. Grimaldi. 1997. Social wasps in amber. American Museum Novitates 3203: 7pp.

Cooper, M. 2000. Five new species of Agelaia Lepeletier (Hym., Vespidae, Polistinae) with a key to members of the genus. New synonymy and notes. Entomologist Monthly Magazine136: 177-198.

Cooper, M. 2001. Two new species of Agelaia Lepeletier (Hym., Vespidae, Polistinae). Entomologist Monthly Magazine137: 233-236.

Jeanne, R. L. 1991. The swarm-founding Polistinae. In Ross, K. G. and R. W. Matthews (eds.), The Social Biology of Wasps: 191-231. Cornell Univ. Press, Ithaca.

Richards, O. W. and M. J. Richards. 1951. Observations on the social wasps of South America (Hymenoptera, Vespidae). Trans. Roy. Entomol. Soc. London 102: 1-170.

Richards, O.W. 1978. Social wasps of the Americas, excluding the Vespinae. London, British Museum (Natural History), 580 p.

Wenzel, J.W. 1998. A generic key to the nests of hornets, yellowhackets and paper wasps worldwide (Vespidae: Vespinae, Polistinae). American Museum Novitates 3224: 1-39.

Zucchi, R.; S. F. Sakagami; F.B. Noll; M.R. Mechi; S. Mateus; M.V. Baio \& S.N. Shima. 1995. Agelaia vicina, a swarm-founding polistine with the largest colony sizy among wasps and bees (Hymenoptera: Vespidae). Journal of the New York Entomological Society103: 129-137. 